\title{
Impact of NBTI on the Performance of 35nm CMOS Digital Circuits
}

\author{
Yangang Wang and M. Zwolinski \\ School of Electronics and Computer Science, University of Southampton, Southampton, SO17 1BJ, UK, \\ E-mails:yw2@ecs.soton.ac.uk,.mz@ecs.soton.ac.uk
}

\begin{abstract}
The negative bias temperature instability (NBTI) of p-MOSFET has the greatest impact on the long term reliability of nano-scale devices and circuits. For several decades, NBTI research has been focused at the device physics level or on the characterization methodology, with little attention paid to the impact of NBTI on the performance of basic digital circuits. This paper discusses the effects of NBTI on $35 \mathrm{~nm}$ technology CMOS inverters and SRAM. The delay degradation and power dissipation of the inverters, as well as the static noise margin degradation of the SRAM are analysed. Moreover, the effects of power supply voltage on inverters and the cell ratio on SRAM under NBTI are also discussed.
\end{abstract}

Keywords: Degradation, inverters, negative bias temperature instability (NBTI), SRAM, static noise margin.

\section{INTRODUCTION}

For state-of-the-art p-MOSFETs, two main factors cause the negative bias temperature instability (NBTI) to restrict device lifetime. One of these is the continuous increase of the effective oxide electric field [1], the other is the adverse effect of introducing more nitrogen into the oxide [2-5]. Furthermore, NBTI was also reported as a critical reliability issue in the high- $\kappa$ dielectric [6]. Therefore, much effort has been devoted to this important reliability issue in the last 10 years. Researchers are usually concerned with the topics such as the physical mechanism of degradation and recovery, as well as the characterization methodology [1-5]. The degradation is generally ascribed to the formation of $\mathrm{Si} / \mathrm{SiO}_{2}$ interface states $\left(\mathrm{N}_{\mathrm{it}}\right)$ and the oxide positive charges $\left(\mathrm{N}_{\mathrm{ot}}\right)$. The $\mathrm{N}_{\mathrm{it}}$ is related to Si dangling bonds because of the breaking of the $\mathrm{Si}-\mathrm{H}$ bonds, and the reaction as well as the $\mathrm{H}$ diffusion was regarded as the mechanism of NBTI kinetics [7]. The $\mathrm{N}_{\mathrm{ot}}$ is linked either with hole trapping by Oxygen/Nitrogen atoms and/or vacancies [2,3], or with the generation process such as the breaking of $\mathrm{Si}-\mathrm{O}$ and $\mathrm{Si}-\mathrm{N}$ bonds [8]. On the other hand, the recovery of NBTI after removing the high stress voltage is firstly thought of as the re-passivation of the $\mathrm{Si}-$ by the returning of $\mathrm{H}$ [9], and then the fast recovery was taken to be the detrapping of trapped holes [10]. Moreover, the traditional measurement technique was thought too slow to monitor the real degradation for losing the fast recovery part. Therefore, some fast techniques were developed which characterize the NBTI by the linear drain current without removing the stress bias, and the degradation can be one order of magnitude higher than that measured by the traditional technique [5,11].

Although NBTI has been considered as one of the design parameters for long term circuit reliability concerns, compared to the research on the above aspects, little work has been reported on the impact of NBTI on nano-scale digital and analog circuits [12-16]. NBTI induces increasing of threshold voltage $\left(\mathrm{V}_{\mathrm{th}}\right)$ and degradation of transconductance, which results in a decrease of the transistor switching speed and the driving capacity. Therefore, the lifetime performance of digital circuit can be affected significantly by NBTI. For analog circuits, problems exist in the NBTI induced mismatch on matched devices pairs [13-15].

In this paper, the effects of p-MOSFET NBTI on the performance of basic nano-scale digital cells, which are the building blocks of most digital circuits, such as CMOS inverters and SRAM are investigated, and a method for mitigating NBTI effects is proposed.

\section{SIMULATION METHODOLOGY}

The performance of the digital cells such as the delays, the power/energy dissipations and the voltage transfer characteristics are simulated by transient and DC analysis using SPICE. The BSIM4 model cards are used with the parameters extracted from $35 \mathrm{~nm}$ technology MOSFETs [17]. The device channel width may change to study the circuit performance variations.

It is reported that the $\Delta \mathrm{V}_{\text {th }}$ of NBTI follows a power law with respect to stress time and the typical $\Delta \mathrm{V}_{\text {th }}$ varies from several tens of millivolts to more than one volt [1-5]. This work does not address the physical origins of NBTI, and the degradations of $\mathrm{V}_{\text {th }}$ are set in the SPICE input files by changing the $\mathrm{V}_{\text {tho }}$ of the p-MOSFET BSIM4 model cards. A series of $\mathrm{V}_{\text {th }}$ are swept one by one during the 
simulation. Unless otherwise specified, the supply voltages $\left(\mathrm{V}_{\mathrm{dd}}\right)$ are $1.0 \mathrm{~V}$, and the circuit temperature is set at $100^{\circ} \mathrm{C}$ which is the typical value for device operational temperature.

\section{RESULTS AND DISCUSSIONS}

\subsection{THE CHAIN OF CMOS INVERTERS}

The performance of a single inverter and a chain of inverters are affected significantly by the $\mathrm{V}_{\text {th }}$ of both $\mathrm{n}$ and p-MOSFETs $\left(\mathrm{V}_{\text {thn }}\right.$ and $\left.\mathrm{V}_{\text {thp }}\right)$. The voltage transfer characteristics (VTC) can drift in parallel under NBTI, which induces variations in the delays, switching characteristics and power dissipations. For nano-scale MOSFETs, the increase in the subthreshold drain current because of short-channel effects also has adverse effects on the performance of inverters.

The inverter delay $\left(t_{d}\right)$, which consists of the high-to-low $\left(\mathrm{t}_{\mathrm{df}}\right)$ and low-to-high $\left(\mathrm{t}_{\mathrm{dr}}\right)$ delays, is related to the switch speed and power dissipation. Therefore, the improvement of $t_{d}$ is always taken into account by designers. In physical terms, $t_{d}$ is determined by the on-state resistance of device as well as by the loading capacitance. For the chain of inverters, the switching delay is characterized by the propagation delay $(\tau)$, which is half of the sum of the pull-down $\tau$ ( $\tau_{\mathrm{n}}$ : time from $\mathrm{V}_{1}=\mathrm{V}_{\mathrm{dd}} / 2$ to $\left.\mathrm{V}_{2}=\mathrm{V}_{\mathrm{dd}} / 2\right)$ and pull-up $\tau\left(\tau_{\mathrm{p}}\right.$ : time from $\mathrm{V}_{2}=\mathrm{V}_{\mathrm{dd}} / 2$ to $\mathrm{V}_{3}=\mathrm{V}_{\mathrm{dd}} / 2$ ) [18]. For ideal cases, the steady state power dissipation $(\mathrm{P})$ is negligible, and $\mathrm{P}$ is controlled by the switching process. However, as the steady state current increases in the nano devices, $\mathrm{P}$ also increases compared to long channel cases.

Fig.1(a) and (b) are the relative delays and power dissipation variations of inverters induced by NBTI. The increase in $\mathrm{V}_{\text {thp }}$ slows the switching speed of the devices, which prolongs the delays. In Fig.1(b), the 5-stage inverters are simulated with all the p-MOSFETs having the same $\Delta \mathrm{V}_{\text {thp }}$

It is reported that the delay of inverters has a power law relationship with stress time, which means the delays increase linearly with $\Delta \mathrm{V}_{\text {thp }}$ [12]. In Fig. 1 , both $\mathrm{t}_{\mathrm{d}}$ and $\tau$ have an approximate linear relationship with $\Delta \mathrm{V}_{\text {thp }}$, which can be explained by the gate delay models [12]. It is noticed that the delay degradation is smaller than the degradation in $\mathrm{V}_{\text {th }}$. When a $100 \mathrm{mV}$ in $\mathrm{V}_{\text {thp }}$ degradation is taken as the failure criteria, the variation ratios of $V_{t h}, t_{d}$ and $\tau$ are $37.1,5.5$ and $16 \%$, respectively, the difference ratios are related to $\mathrm{V}_{\mathrm{g}}-\mathrm{V}_{\text {th }}$ in theory [12].
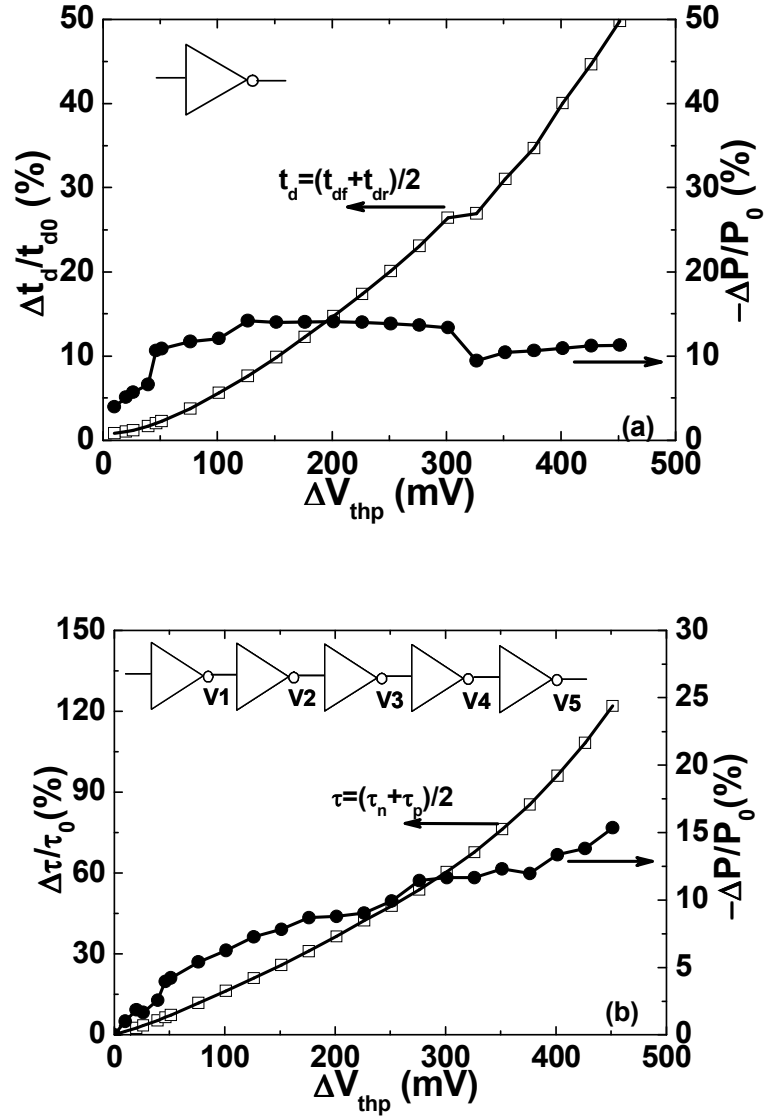

Fig.1 The delays and power dissipations of the inverter (a) and the chain of inverters (b) under NBTI

The power dissipation is another limiting factor for designing nano-scale circuits. The increase of delays because of NBTI does not cause an increase in the power/energy dissipations. On the contrary, as shown in the Fig.1, P decreases significantly under NBTI. The reason is that both the subthreshold drain current (steady state current) and the switching current decrease significantly with $\mathrm{V}_{\text {thp }}$ increasing.

With the scaling down of the device dimensions, the power-supply voltage decreases, which induces an increase in the delays but a decrease of P. Fig. 2 shows the relative variations of $\tau$ and $P$ with $V_{d d}$ decreasing under NBTI. The delay degradations are enhanced by lower $V_{d d}$, while the $P$ improvements are insensitive to $\mathrm{V}_{\mathrm{dd}}$ to some extent. According to Fig.2, it is suggested the trade-off $\mathrm{V}_{\mathrm{dd}}$ for the $35 \mathrm{~nm}$ CMOS inverters subjected to NBTI is $0.9-1.0 \mathrm{~V}$. 


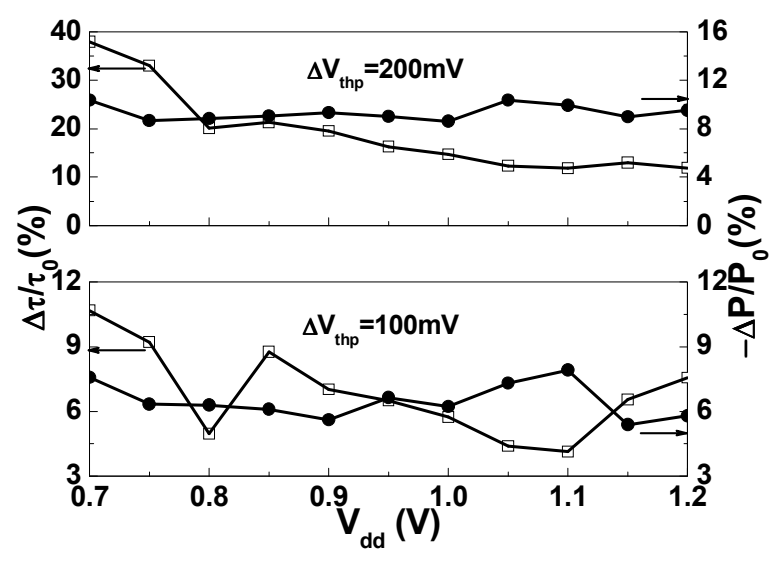

Fig. 2 The $\tau$ and $P$ variations of the chain of inverters under NBTI versus the power-supply voltage

\subsection{THE CMOS SRAM}

As one of the most important parts of modern microprocessors and SOCs, the SRAM cell is often considered as the benchmark for the development of CMOS technology $[17,19]$. $\mathrm{V}_{\text {th }}$ mismatch will result in stability problems for SRAM using minimum geometry transistors. The stability of an SRAM cell is always characterized by the static noise margin (SNM), which is defined as the minimum DC noise voltage needed to flip the cell state $[17,19]$. SNM is a limiting factor for trading off the performance of a technology against the cost. The SNM can be calculated by the smaller area formed by the two static transfer characteristic (STC) curves. For the symmetric SRAM cell structure illustrated in Fig.3, the STCs are almost the same by either sweeping the $\mathrm{V}_{\mathrm{SL}}$ or the $\mathrm{V}_{\mathrm{SR}}$.

Therefore, it is crucial to investigate the NBTI of SRAM for the moment [20]. The STC and SNM definition for a SRAM cell at initial state and at $\Delta \mathrm{V}_{\text {thp }}=200 \mathrm{mV}$ are shown in Fig.3, the inset has the circuit schematics of a traditional SRAM cell. The two p-MOSFETs (M1, M3) have the same $\Delta \mathrm{V}_{\text {thp }}$ during the simulation. The $\Delta \mathrm{V}_{\text {th }}$ can affect the shapes of the STCs and then the SNM.

Fig. 4 shows the relative degradation of SNM as a

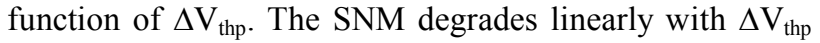
(i.e. the stress time power law), which will in return limit the lifetime of the circuits. It is reported that the SNM degradation increases with decreasing supply voltage [21], so the importance of NBTI on SRAM will be more significant with the scaling of the device dimensions.

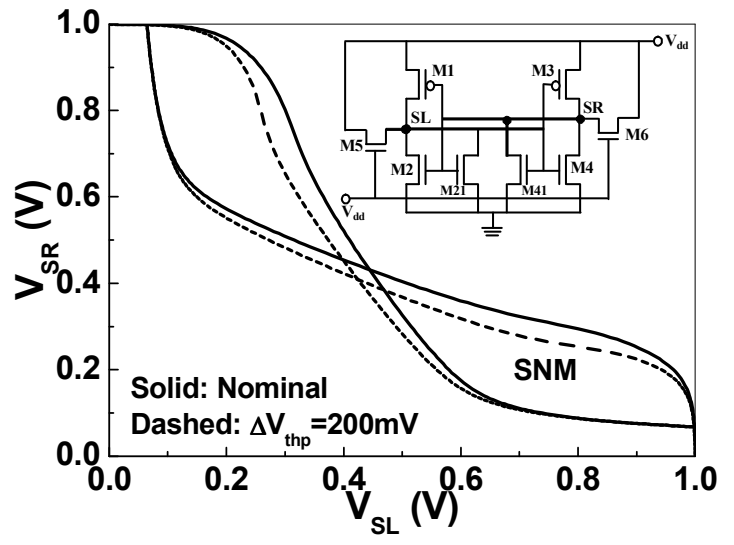

Fig. 3 The static transfer characteristics and the static noise margin of the SRAM, inset is the circuit schematics

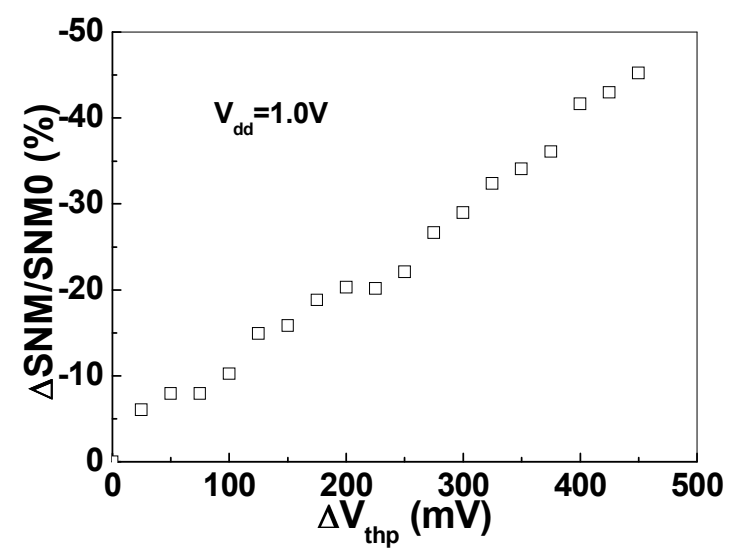

Fig.4 The relative degradation of SNM under NBTI

It is believed that the stability of SRAM can be improved by increasing the cell ratio (CR), which is the ratio of the driver transistor's (M2, M4) width/length (W/L) to the access transistor's (M1, M3) W/L [17,22]. During the above simulation, the CR is set to 2 . When the CR increase to 5, Fig.5(a) shows the improvement of SNM is about $22 \%$ compared to $\mathrm{CR}$ equals to 2 for a nominal SRAM cell. Moreover, as shown in Fig.5(b), the SNM degradation ratios always reduce because of CR increasing at different levels of NBTI. However, the improvement of relative SNM degradation is not significant when CR become more than 3. As a large $\mathrm{CR}$ results in the increasing of cost and high power/energy dissipations, designers should take the above into account to find the optimal designs. 


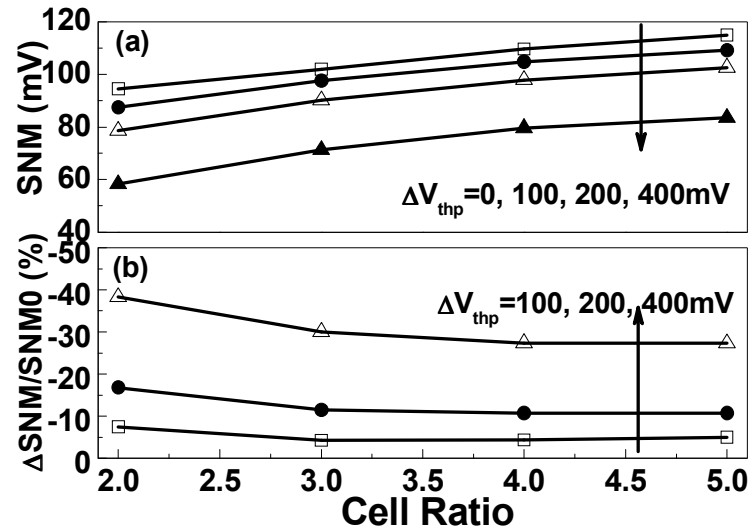

Fig.5 Both the SNM (a) and the relative degradation of SNM (b) are improved by the increasing of the cell ratio

\section{CONCLUSIONS}

The effects of NBTI on the performance of the $35 \mathrm{~nm}$ CMOS inverters and SRAM are simulated in this paper. Although the delay degradation of the inverters increases significantly with NBTI, the power dissipation also decreases to some extent. The increasing of delays is induced by gate delays, and the decreasing of power dissipation results from the reduction of the subthreshold drain current and switching current. When taking the effects of NBTI on the delays and power dissipations into account, the trade-off $\mathrm{V}_{\mathrm{dd}}$ for the $35 \mathrm{~nm}$ CMOS inverters is $0.9-1.0 \mathrm{~V}$. On the other hand, for the traditional SRAM cells, the static noise margin degrades linearly with the NBTI. However, the SNM and the relative SNM degradation can be improved by increasing the cell ratio with a relatively higher cost. It is suggested the optimal cell ratio for the $35 \mathrm{~nm}$ CMOS SRAM is about 3 .

\section{ACKNOWLEDGEMENTS}

This work is supported by the Engineering and Physical Sciences Research Council (EPSRC) of the United Kingdom under the grant No. EP/E002064/1.

The authors are grateful for supplying the Model Cards by the Device Modelling Group, University of Glasgow.

\section{REFERENCES}

[1] D. K. Schroder and E. H. Nicollian, J. Appl. Phys., 94, p.1 (2003)

[2] S. S. Tan, T. P. Chen and C. H. Ang et al., IEEE Electron Device Letts., 25, p.504 (2004)

[3] J. Ushio, T. Maruizumi and K. K-Abdelghafar, Appl. Phys. Letts., 81, p.1818 (2002)

[4] B. Kaczer, V. Arkhipov and R. Degraeve et al., Appl. Phys. Letts., 86, art. no.143506 (2005)

[5] M. Denais, A. Bravaix and V. Huard et al., IEEE IEDM, p.109 (2004)

[6] A. S. Oates, IEEE IEDM, p.923 (2003)

[7] M. A. Alam and S. Mahapatra, Microelectron. Reliab., 45, p.71 (2005)

[8] D. Varghese, S. Mahapatra, and M. A. Alam, IEEE Electron Device Lett., 26, p.572 (2005)

[9] G. Chen, M. F. Li and C. H. Ang et al., IEEE Electron Device Lett., 23, p.734 (2002)

[10] T. Yang, M. F. Li and C. Shen et al., IEEE VLSI Symp. Tech. Dig., p.92 (2005)

[11] K. Kang, M. A. Alam and K. Roy, IEEE IRPS, p.16 (2007)

[12] B. C. Paul, K. Kang and H. Kufluoglu, et al., IEEE Electron Device Letts., 26, p.560 (2005)

[13] N. K. Jha, P. S. Reddy and D. K. Sharma et al., IEEE Trans. Electron Devices, 52, p.2609 (2005)

[14] X. N. Yang, E. Weglarz and K. Saluja, IEEE VLSID, p.723 (2007)

[15] M. Agostinelli, S. Lau and S. Pae et al., Microelectron. Reliab., 46, p.63 (2006)

[16] B .C. Paul, K, Kang and H. Kufluoglu et al., IEEE IEEE Trans. Comput-Aided Design Integr. Circuits Syst. 26, p.743 (2007)

[17] B. Cheng, S. Roy and G. Roy et al., Solid State Electron., 49, p.740 (2005)

[18] Y. Taur and T. H. Ning, Fundamentals of Modern VLSI Devices, Cambridge University Press, p.258 (1998)

[19] A. J. Bhavnagarwala, X. H. Tang, and J. D. Meindl, IEEE J. Solid-State Circuits, 36, p.658 (2001)

[20] K. Kang, S. P. Park and K. Roy, in IEEE/ACM ICCAD, p.730 (2007)

[21] V. Reddy, A. T. Krishnan and A. Marshall et al., Microelectron. Reliab., 45, p.31 (2005)

[22] B. Cheng, S. Roy and A. Asenov, Solid State Electron., 51, p.565 (2007) 